\title{
TRANSMEMBRANE SIGNALLING PATHWAYS CONTROLLING CELL GROWTH
}

\author{
Pouysségur, J. \\ Centre de Biochimie-CNRS, Parc Valrose, 06034 NICE CEDEX - FRANCE
}

A wide variety of growth promoting agents including polypeptide growth factors, hormones, neurotransmitters. vasoactive agents stimulate a common pleiotypic response which precedes the onset of DNA replication. A major question still poorly uderstood is how these various mitogens transduce their signal across the plasma membrane? Which transmembrane signalling pathway play a determinant role in initiating or preventing G0 exit? With a model of hamster lung fibroblasts in culture capable of reversible growth arrest in GO and responding to $\alpha$ thrombin, EGF,FGF, IGF-I, serotonin, we were able to identify three major and independent signalling pathways, two initiating cell growth and one preventing GO exit.

1) The first transducing mechanism elicited by thrombin, bombesin, bradykinin, vasopressin, serotonin ... is mediated through G-protein activation. At least two $G$-proteins are involved in this action:

- Gp, a G-protein which activates phospholipase C

- Gi's. pertussis toxin-sensitive substrates coupled negatively to adenyl cyclase and other effectors.

2) The second transducing mechanism elicited by EGF, PDGF, CSF-I, FGF and IGF-I is strictly mediated through tyrosine kinase activation and is independent of G-protein-mediated processes.

Both signalling pathways could act independently or synergistically to initiate cell growth.

3) The third pathway preventing GO exit is elicited by any hormone activating Gs, the G-protein coupled positively to adenylcyclase. This antimitogenic action is exerted only at the early stages of GO exit.

Finally, we shall present and discuss new molecular approaches to dissect further the multiple G-protein activated pathways and precise their role in controlling cell growth.

EFFE'T DE L'OESTRADIOL, DE L'EGE ET DE L'INSULINE SUR LA PROLIFERATION DES CELLULES EPITHELIALES DE L'ENDOMETRE CULTIVEES EN MILIEU DEFINI

Adessi G.L. et Alkhalaf M.

INSERM U 198,25000 BESANCON

Le mécanisme d'action des oestrogènes sur la prolifération des cellules normales ou cancéreuses n'est pas encore élucidé. In vivo, l'oestradiol-17ß a une action indiscutable sur la prolifération des cellules utérines, vaginales et de la glande mammaire. Par contre, in vitro, son action sur les cellules normales ou transformées ne serait pas directe mais indirecte (autocrine et/ou paracrine). L'action de l'oestradiol-17ß, de l'EGF et de l'insuline sur la synthèse de l'ADN et la prolifération cellulaire a été étudiée in vitro sur des cellules epithéliales de l'endomètre. Pour cela, les cellules épithéliales ont été cultivées et synchronisées en phase $G_{0} / G_{1}$ par privation nutritive en milieu défini. Dans ces conditions, l'oestradiol $(0.1$ à $1000 \mathrm{nmol} / 1) \mathrm{n}$ 'induit pas la prolifération cellulaire mais a une action inhibitrice. Au contraire, le sérum de veau foetal désactivé et déstérö̈dé, l'EGF ou l'insuline provoquent le passage des cellules en phase S. L'oestradiol-17ß ne potentialise pas l'action de l'EGF ni de l'insuline mais augmente l'action mitogène du sérum de veau traité.

Au total, in vitro, l'oestradiol-17ß n'a pas d'action mitogène directe mais il potentialise l'action du sérum par l'intermédiaire d'un ou plusieurs facteurs qui ne sont pas apparentés à l'EGF ou au facteurs de croissance liés à l'insuline. 